\title{
GMNF-DVMRP : A Modified Version of Distance Vector Multicast Routing Protocol
}

\author{
Yuan-Cheng Lai Ying-Dar Lin Wei-Che Yu Yuh-Tay Lin \\ Department of Computer and Information Science \\ National Chiao Tung University \\ 1001 Ta Hsuch Rd., Hsinchu, Taiwan 30050, R.O.C.
}

\begin{abstract}
In this paper, we make a survey of Distance Vector Multicast Routing Protocol (DVMRP) and find that it is not optimal in the aspect of network cost (NC). Therefore, we propose a modified version of DVMRP, called "Group Membership Near First-DVMRP" (GMNF-DVMRP), to decrease the NC of the multicast tree formed by DVMRP. A simulation is implemented to compare our modified version and the original DVMRP. We find that our method save about 5-7 percent of NC of the multicast tree formed by DVMRP.
\end{abstract}

\section{1: Introduction}

A multicast routing protocol is a set of standards and parameters that two end points of communication agree upon to route packets. The Internet Group Management Protocol(IGMP)[1] is used to carry messages between the multicast router and the end-host or between multicast routers themselves. A variety of multicast routing schemes have been developed and used. They include Distance Vector Multicast Routing Protocol(DVMRP)[2] Core-Based Trees multicast protocol (CBT)[3] and Protocol Independent Multicast protocol(PIM)-Dense Mode and Sparse Mode[4]. A simple comparison of these schemes is given in Table 1. However, we lay our focus on DVMRP because it has been implemented in the mrouted program.

Two optimality criteria are used to evaluate the efficiency of the multicast tree formed by a multicast routing protocol, namely network $\operatorname{cost}(\mathrm{NC})$ and destination $\operatorname{cost}(\mathrm{DC})[5] . \mathrm{NC}$ is a value that measures the utilization of the network resources, such as total used bandwidth. DC is a value that measures the average delay experienced by each destination. Minimizing DC is simpler than minimizing $\mathrm{NC}$, because finding the minimum $\mathrm{NC}$ multicast tree on a given network topology is an NP- complete problem--also called a minimal Steiner tree problem[6].

The DC of the DVMRP multicast tree is optimal because it is built on the shortest paths from the source to each destination. However, the NC of the DVMRP multicast tree may be high or low, depending on the network topology and the distribution of group members. Shared medium networks, especially the Internet, have sensitive NCs. Redundant use of one hop on the multicast tree will cost much bandwidth, especially when the volume of multicast data is large. Therefore, we should try to decrease the NCs of multicast trees formed by the multicast routing protocol. In this paper, we developed a new version of DVMRP as GMNF-DVMRP to decrease the NC.

In the following sections, we propose a modified version to improve DVMRP in section 2. A simulation that compares DVMRP and our modified version is presented in section 3. Finally, a conclusion is given in section 4 .

\begin{tabular}{|l|c|c|c|c|c|}
\hline Protocol & $\begin{array}{c}\text { Tree } \\
\text { Type }\end{array}$ & $\begin{array}{c}\text { Group } \\
\text { Distribution }\end{array}$ & $\begin{array}{c}\text { Protocol } \\
\text { Independence }\end{array}$ & $\begin{array}{c}\text { State } \\
\text { Mechanism }\end{array}$ & $\begin{array}{c}\text { Scalabil } \\
\text { ity }\end{array}$ \\
\hline DVMRP & $\begin{array}{l}\text { source } \\
\text {-based }\end{array}$ & dense & NO & hard & Bad \\
\hline CBT & $\begin{array}{l}\text { shared } \\
\text {-based }\end{array}$ & sparse & NO & hard & Good \\
\hline PIM-DM & $\begin{array}{l}\text { source } \\
\text {-based }\end{array}$ & dense & YES & soft & Bad \\
\hline PIM-SM & mixed & sparse & YES & soft & Good \\
\hline
\end{tabular}

Table 1: A simple comparison of multicast routing schemes.

\section{Modified Version of DVMRP}

\section{1: An example}

Consider an example of building a multicast tree of 
DVMRP on the network topology illustrated in figure 1. Multicast packets are sent from the source to multicast router MR1. MR1, the designated multicast router of subnetwork $\mathrm{S} 2$ and $\mathrm{S} 3$, then delivers these multicast packets onto S1 and S2. Assume that the addresses of MR2 and MR3 are 140.113 .136 .110 and 140.113.136.111, respectively. MR2 becomes the designated multicast router of subnetwork S2 with a lower address than MR3, though they have the same distance of 2 hops to the source As a result, MR2 is responsible for delivering these milicast packets onto S1 but MR3 is not MR3 will send a prune message back to its predecessor MR1. We consider NC as the total hop number used by the multicast tree. Thus, the NC of this multicast tree is 4 . If, however, MR3 wins to be the designated multicast router, $\mathrm{MR} 3$ will be responsible for delivering multicast packets onto $\mathrm{S} 4$ rather than sending a prune message back On the contrary, MR2 will send a prune message to its predecessor MR1 and MR1 will then stop delivering multicast packets onto $\mathrm{S} 2$. The NC of the new multicast tree is 3, resulting in one hop saving. Since MR2 and MR3 own the same distance to the source, the DC of the new multicast tree is equivalent to that of the previous multicast tree. The new multicast tree is shown in figure 2 .

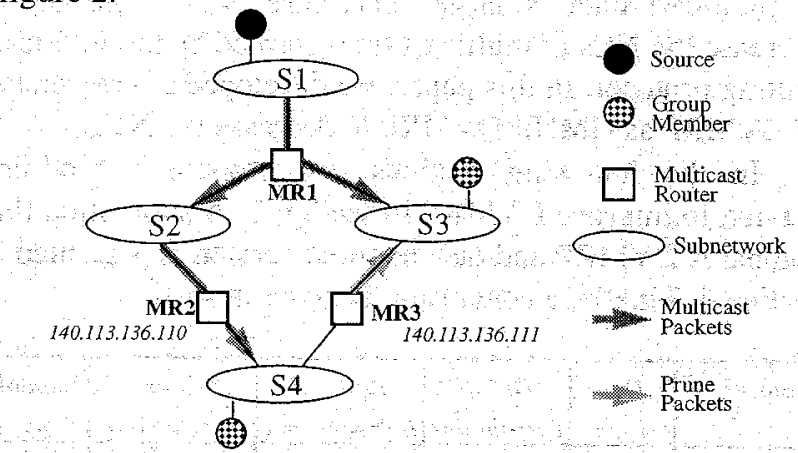

Figure 1. An example of building multicast tree of DVMRP where MR2 is the designated router of $\$ 4$.

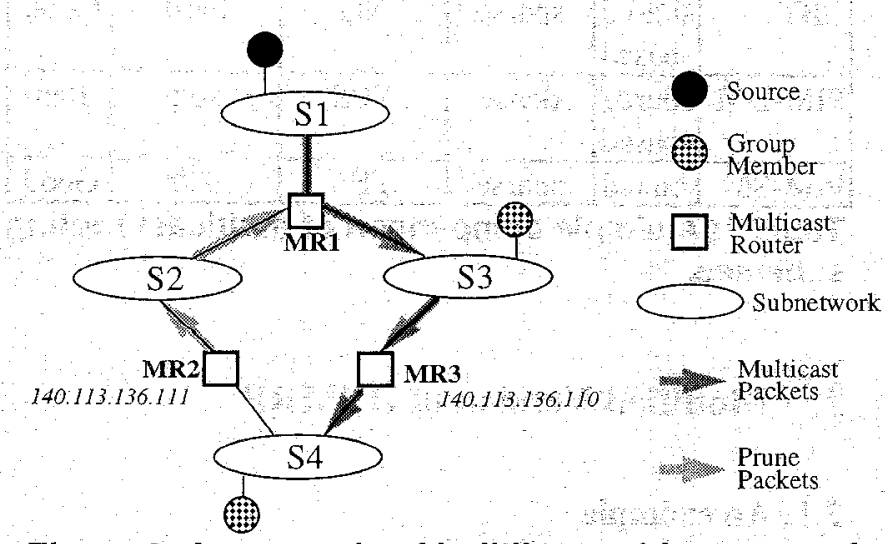

Figure 2: An example of building multicast tree of DVMRP where MR3 is the designated router of S4.

\section{2: Idea}

The idea of our modified version of DVMRP is simple. If two or more shortest paths exist between a source and its destination, choose the path where the first predecessor has one group member. Whenever a host joins a group, the interrogating multicast router of the host(MR_A) sends this group address to its neighboring multicast routers(MR_B). Multicast router MR B now knows that a member of such group address exists on the subnetwork of MR A. From now on, whenever MR $B$ receives multicast packets, it checks if the multicast address of these packets is the group address it received from its neighboring multicast routers, for example MR A. If it matches, then $G M N$ (source address) of $\mathrm{MR} \mathrm{B}$ is set to be true, where source address is the source address of these multicast packets. GMN(source address) means that group membership of the sender with source address exists on the neighboring subnetwork. Now MR B has higher priority to become the designated multicast router under the situation that other multicast routers on the same subnetwork have the same shortest distance to this source. Consider the example shown in figure 1 again. MR1, the interrogating multicast router of $\mathrm{S} 3$, sends a message to MR3 to announce that a member of a particular group address exists on $\mathrm{S} 3$. When MR3 receive multicast packets of such group address, it retrieve the source address from the header of the first packets and set GMN(source address) to be true. Now MR3 has higher priority to become the designated multicast router for forwarding multieast packets from that source although its address is not the lowest.

\section{3: Algorithm}

We call our modified version of DVMRP as GMNFDVMRP. GMNF is short for GGroup Membership Near First". It means that if there is a member on the neighboring network, the algorithm should consider the router attached to this neighboring subnetwork first to become the designated multicast router of its subnetwork.

Each interrogating multicast router of a subnetwork sends the message of group membership to its neighboring multicast routers periodically. The packet format of the message is shown as the following:

\begin{tabular}{|c|c|c|}
\hline multicast group & $\begin{array}{r}\text { multicast group } \\
\text { address1 }\end{array}$ & address2 \\
\hline
\end{tabular}

After receiving this message, the receiving multicast router will record these multicast group addresses in a table. Whenever multicast packets from a particular source come, this multicast router will compare the multicast address in the packet header with those in the small table. If the multicast address of the incoming packets exists in the 
small table, GMN(the source address) of the multicast router is set to be true. The DVMRP algorithm is modified as follows:

WHILE $R$ receives "routing updates" entry (d, D, next-hop-address) from $n$

IF (next-hop-address $<>$ k) AND

(NO n such that (n.subnet=k AND

(n.distance $[\mathrm{d}]<\mathrm{D}$ OR

(n.distance $[d]=D A N D$ (NO R.GMN(d) AND n.GMN(d) OR

(R.GMN(d)AND n.GMN(d) AND n.address on $k<$ R.address)))))

THEN $\mathbf{k}$ is a child of $\mathrm{R}$ on the multicast tree rooted at $d$

IF (NO $n$ that ( $\mathrm{n}$.subnet=k and $\mathrm{n}$.distance[d]=infinity))

THEN $k$ is a leaf of $R$ on the multicast tree rooted at $d$

\section{Procedure 1: Modified version of DVMRP.}

\section{3: Simulation}

We have written programs to simulate the above two algorithms. We measure the NC of a multicast tree in a simple way, namely, by its total hop counts.

\section{1: Network model}

We model the network as an undirected graph where nodes represent multicast routers and edges represent links or subnetworks connecting routers. Precisely speaking, a node represents a multicast router and its connected hosts. A node, where one of its connected hosts is the sender of multicast packets, is the source and a set of nodes, where one or more of its connected hosts are the receiver of multicast packets, excluding the source node are group members.

To create such a network with $\mathrm{N}$ nodes, each node is assigned a random coordinate in the unit square. The edge between two nodes is generated depending on the probability function of its length. We adopt the following probability distribution function described in Ken Calvert's Technical report[7] to generate edges:

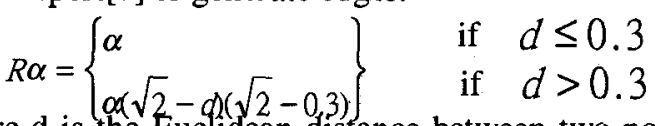

where $d$ is the Euclidean distance between two nodes, and $\alpha$ is a constant that affects the average degree of the graph. If the distance between two nodes is no more than 0.3 unit, the probability to generate an edge between these two nodes is fixed to be $\alpha$. If the distance is more than 0.3 , the probability decreases linearly with the distance. In order not to create any isolated node in the graph, we connect nodes one by one at the beginning, that is, node 1 to node2, node2 to node3, and so on. An example of such a graph with 100 nodes is illustrated in figure 3 .

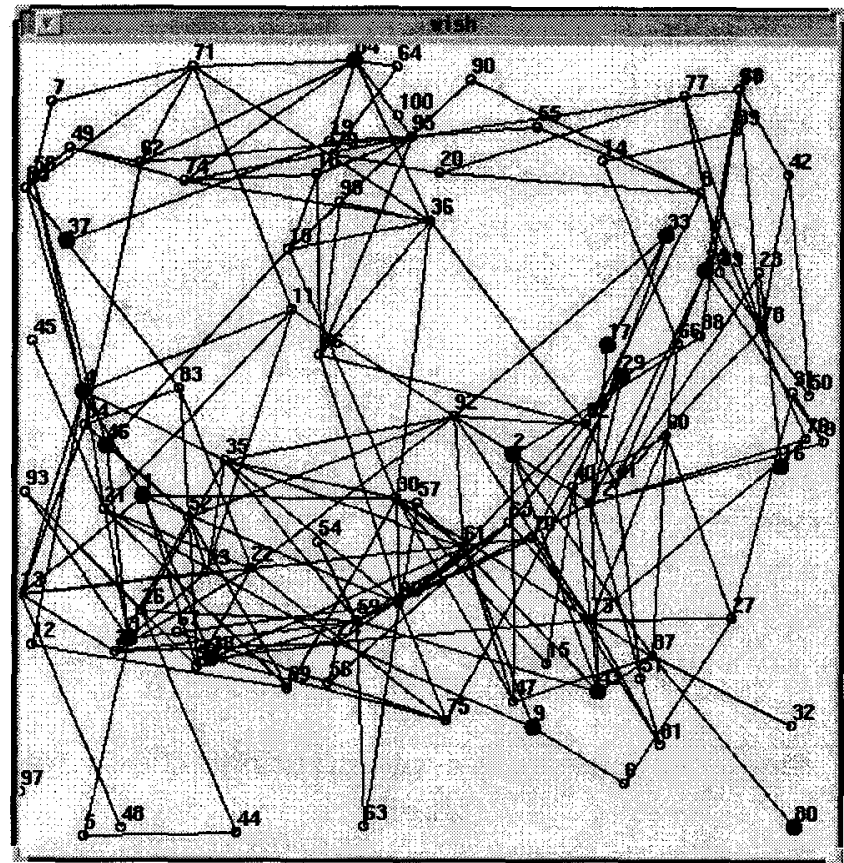

Figure 3: An example of 100 nodes graph.

\section{2: Simulation result}

Two combinations of size and connectivity is used: a 400-node graph with an average degree of 5.0375 and a 900 -node graph with an average degree of 3.47 . We generated 10 graphs of each combination.

The simulation results of the two multicast algorithms under these two combinations are shown in figure 4, 5, 6, and figure 7. Our GMNF-DVMRP algorithm has the best effect when the number of receiver nodes is about half of the total node number. It decreases about 7 percent of $\mathrm{NC}$ in the 900 -node graph. Although 7 percent is a small number, saving one hop on a multicast tree may result in saving lots of network bandwidth because the volume of multicast packets is large.

\section{4: Conclusion}

We have made a survey of DVMRP and found that it is not optimal in the aspect of NC(network cost). Therefore, we propose a modified method of DVMRP, called GMNF DVMRP, to decrease the NC of the multicast tree formed by DVMRP. A simulation has been implemented to compare our modified method and the original DVMRP. We find that our method save about 5-7 percent of $\mathrm{NC}$ of the multicast tree formed by DVMRP.

In this paper, we lay emphasis on DVMRP because it is implemented in the mrouted program. However, DVMRP is considered as the first generation multicast routing protocol. 
Other multicast protocols have been developed and considered to be better than the DVMRP. For future research, we are going to study these second generation multicast protocols and try to find a better solution.

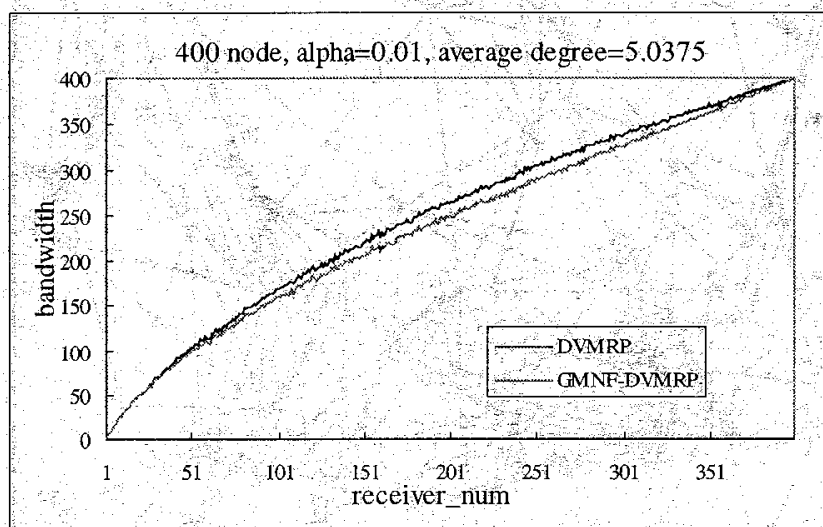

Figure 4: Bandwidth consumption in 400-node graphs.

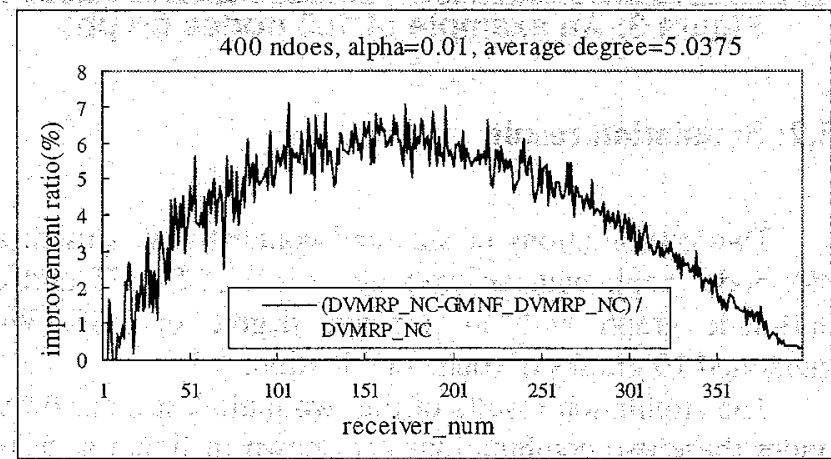

Figure 5: Improvement ratio of bandwidth in $\mathbf{4 0 0}$ node-graphs.

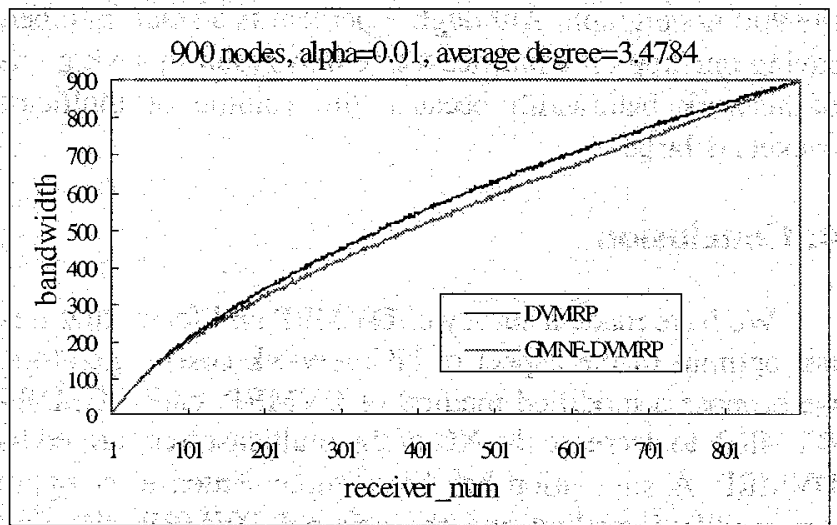

Figure 6: Bandwidth consumption in 900 -node graphs.

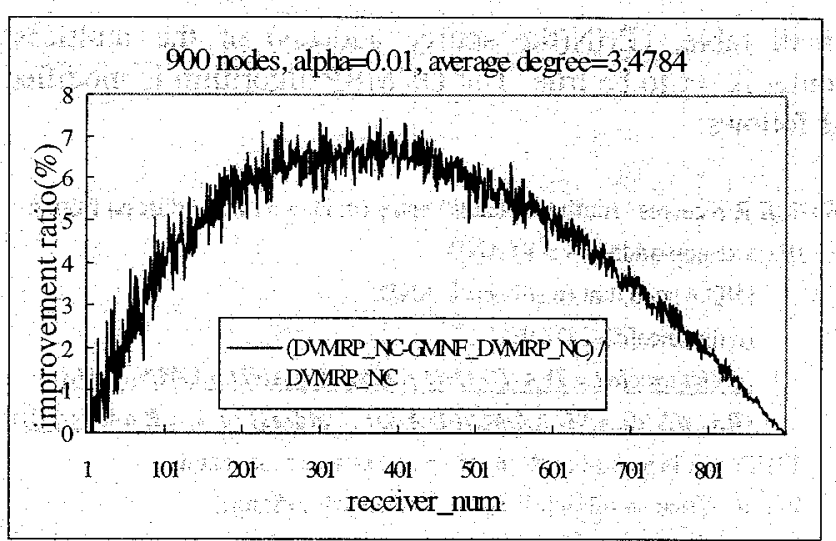

Figure 7: Improvement ratio of bandwidth in 900node graphs.

\section{References}

[1]S. Deering, "Host Extensions for IP Multicasting, RFC 1112, August $1989, \quad$ http $/$ www cis ohiostate.edu/htbin/rfc/rfcl 1 12. html.

[2]Waitzman, D., C. Partridge, and S. Deering, "Distance Vector Multicasting Routing Protocol," Network Workgroup Request for Comments, Internet RFC-1075, November 1988, ftp://ftp.ietf.cnri.reston.vä.us/internet-drafts/draft-ietf-idmrdvmrp-v3-00.txt.

[3]Stuart Aaren, "Managing ATM Virtual LAN Workgroups," Newbridge Networks Inc Tecknical Document, 1995, http /www vivid newbridge.com/documents/, vividmanaging.html:

[4]Jean Quale, "Building Scalable ATM LANs," Newbridge Networks Inc. Technical Document, April 1994, http //www vivid newbridge.com/documents/ vividscalable.html.

[5]Kadaba Bharath-kumar and Jeffrey M. Jaffe, "Routing to Multiple Destinations in Computer Networks," IEEE Transactions on Communication, March 1983, Vol Com-31. No.3.

[6]E. N. Gilbert and H. O. Pollak, "Steiner minimal tree", SLAM Journal of Applied Math., Vol. 16, 1968.

[7]Ken Calvert, Ramesh Madhavan, and Ellen W.Zegura, "A Comparison of Two Practical Multicast Routing Schemes," College of Computing, Georgia Institute of Technology, GITCC-94/25, Feb 1994. 